\title{
A rare case of carbimazole induced severe aplastic anemia with fatal outcome
}

\author{
Naveen L*, Santoshi Malkarnekar, Prathima S \\ MD Medicine, Sri Devaraj Urs Medical College, Kolar, India \\ MD Pathology, Sri Devaraj Urs Medical College, Kolar, India \\ *Corresponding author E-mail: drnaveenkgf@gmail.com
}

\begin{abstract}
Though agranulocytosis is a well known adverse effect of antithyroid drugs (ATDs), aplastic anemia is thought to be rare. Herein we present a case report of carbimazole induced aplastic anemia in a patient of Grave's disease, who satisfied all the criteria for severe disease and had profound bone marrow hypoplasia. Drug induced aplastic anemia is thought to be a result of an idiosyncratic response directed against hematopoietic stem cells and is managed in a similar fashion to idiopathic aplastic anemia. The hematopoietic damage in our patient did not recover following ATD withdrawal and supportive treatment. This necessitated the use of immunosuppressive therapy with cyclosporine and antithymocyte globulin, which she could not afford and despite of all the supportive treatment she eventually succumbed to severe sepsis. Since ATDs are commonly used in clinical practice, the physicians should be aware of this rare but lifethreatening complication.
\end{abstract}

Keywords: Antithyroid drugs (ATDs), Aplastic anemia, Carbimazole, Grave's disease, Pancytopenia.

\section{Introduction}

Antithyroid drugs (ATDs) have been used as a standard therapy for Grave's disease for more than 50 years. Though well tolerated, ATDs may cause serious life threatening side effects such as agranulocytosis and aplastic anemia. ATDs induced aplastic anemia is a very rare event (Richardson et al. 1954, Biswas et al. 1991, Escobar-Morreale et al. 1997). And hence we report one such case at our centre.

\section{Case report}

A 50 year old lady presented with complaints of easy fatiguibility, easy bruisablity and bleeding gums for 15 days. She is a known case of Grave's disease on treatment with carbimazole $(10 \mathrm{mg}$ thrice a day) for the past three months. Physical examination revealed pale skin and mucous membranes, bleeding gums, ecchymotic patches on legs and body. There was no lymphadenopathy and hepato-splenomegaly, other systems being unremarkable. At admission, her blood film revealed pancytopenia with $2.6 \mathrm{gm} / \mathrm{dl}$ haemoglobin concentration, $0.5 \times 10^{9} / \mathrm{L}$ leukocytes and severe thrombocytopenia of $2.0 \times 10^{9} / \mathrm{L}$. Differential count showed $91 \%$ of lymphocytes, $1 \%$ of monocytes and $8 \%$ of neutrophils. The peripheral smear showed normocytic normochromic picture and absence of blast cells. Absolute reticulocyte count was $20 \times 10^{9} / \mathrm{L}$. Bone marrow biopsy revealed severe hypocellularity, replaced with fat cells, iron stores being normal, as shown in Figure. 1. Other tests done such as antinuclear antibody, coombs test, serum iron and cobalamin levels, abdominal ultrasonography, chest radi- ography, renal and liver function tests, were within normal limits. Review of the past records revealed a normal hemogram before starting therapy with ATDs and a thyroid profile consistent with thyrotoxicosis (TSH- $0.001 \mathrm{mcIU} / \mathrm{ml}, \mathrm{T} 4-17.6 \mathrm{mcg} / \mathrm{dl}$ and T3$365 \mathrm{ng} / \mathrm{dl}$ ). She was clinically and biochemically euthyroid during her present hospitalization. Her antithyroid therapy was discontinued and supportive treatment in the form of packed cell and platelet transfusions, colony stimulating factors, prednisolone and barrier nursing was started. No significant hematological recovery was noted and in view of this therapeutic failure she was advised immunosuppression therapy with cyclosporine and antithymocyte globulin, which she refused due to financial constraints. At the end of one month, she was discharged from the hospital at her request. She was counselled regarding her condition and the need for regular follow up. At the time of discharge her hemogram revealed $6.0 \mathrm{gm} / \mathrm{dl}$ hemoglobin concentration, $2.00 \times 10^{9} / \mathrm{L}$ leucocytes and platelets of $40 \times 10^{9} / \mathrm{L}$. Differential count showed $78 \%$ of lymphocytes, $2 \%$ of monocytes and $20 \%$ of neutrophils. She followed up regularly at the outpatient department with intermittent hospitalizations for blood transfusions for the next two months, after which she was lost for follow up. One month later patient presented to the emergency room with complaints of fever for three days. On examination, she had pale mucous membranes, bleeding gums, generalized ecchymosis. She had a feeble pulse, blood pressure of $70 / 50 \mathrm{~mm} \mathrm{Hg}$ and cold clammy extremities. Breath sounds were normal on auscultation. Hemogram revealed $2.5 \mathrm{gm} / \mathrm{dl}$ hemoglobin concentration, $0.4 \times 10^{9} / \mathrm{L}$ leucocytes and severe thrombocytopenia of $2.0 \times 10^{9} / \mathrm{L}$. Differential count showed $94 \%$ of lymphocytes, $3 \%$ of monocytes and only $3 \%$ of neutrophils. Electrocar- 
diogram and chest radiograph were within normal limits. Blood cultures were drawn and broad spectrum antibiotics were started. She was given packed cell and platelet concentrate transfusions, colony stimulating growth factors along with other necessary supportive care. Despite vigorous fluid rescusitation, her shock did not improve, which necessitated the use of inotropes. She eventually succumbed to severe sepsis within 48 hours of admission.

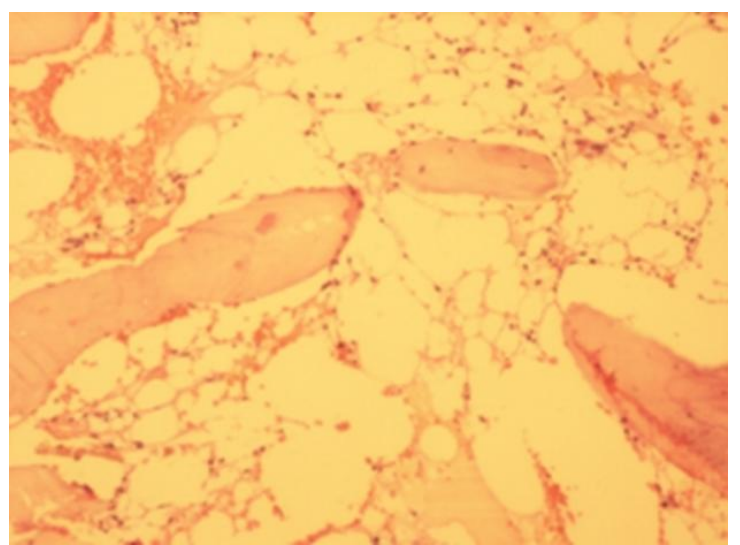

Fig. 1: Bone marrow showing marked hypocellularity and replacement by fat cells.

\section{Discussion}

Aplastic anemia was first recognized by Ehrlich in 1888. Acquired aplastic anemia is a clinical syndrome in which there is a deficiency of red cells, neutrophils, monocytes and platelets in the blood, and fatty replacement of the marrow with a near absence of hematopoietic precursor cells. The diagnosis usually requires the presence of pancytopenia with neutrophil count fewer than $1.5 \mathrm{x}$ $10^{9} / \mathrm{L}$, a platelet count less than $50 \times 10^{9} / \mathrm{L}$, a hemoglobin concentration less than $10 \mathrm{gm} / \mathrm{dl}$, an absolute reticulocyte count fewer than $40 \times 10^{9} / \mathrm{L}$, accompanied by a hypocellular marrow without abnormal or malignant cells or fibrosis ( Blood 1987, p. 1718). A bone marrow cellularity of less than $25 \%$ and marked decreased values of at least two of three hematopoietic lineages (neutrophil count less than 500/microliter, platelet count less than $20,000 /$ microliter and absolute reticulocyte count of less than $60,000 /$ microliter) defines severe aplastic anemia, as seen in our patient.

Though most of the cases are idiopathic, a plethora of drugs have been implicated as a cause of aplastic anemia. Whether idiopathic or associated with an inciting agent, such as a drug, the reduced hematopoiesis in aplastic anemia results from cytotoxic T-cell mediated immune suppression of very early CD34+ hematopoietic multipotent progenitor or stem cells (Kumar \& Goldman 2002).

Occasional cases of aplastic anemia are seen in conjunction with autoimmune diseases. In vitro studies have found either the presence of an antibody or suppressor cell directed against hematopoietic progenitor cells. Severe aplastic anemia also has been reported co-incident with immune thyroid diseases such as Grave's disease and the aplasia has been reversed with treatment of hyperthyroidism (Young et al. 2006, Aydin et al. 2008). However this seems to be an unlikely association in our patient, since she was clinically and biochemically euthyroid at the time of diagnosis of aplastic anemia.

When drugs are implicated in causing aplastic anemia, it is important to recognize that unlike agranulocytosis and drug induced thrombocytopenia, stopping the offending drug does not lead to hematopoietic recovery (Williams 1999, p. 1186-1187). Most cases of drug- induced aplastic anemia lead to an idiosyncratic immune response directed against hematopoietic stem cells and are managed similarly to those with idiopathic aplastic anemia. The same was the case with our patient, in whom the hematopoietic damage did not improve following drug withdrawal and our patient eventually succumbed to her illness.

\section{Conclusion}

This case report emphasizes that ATDs may cause hematopoietic changes, which can be fatal. Since ATDs are commonly used in clinical practice, the physicians should be aware of this rare but life threatening complication.

\section{Acknowledgement}

Authors express gratitude to R.L .Jallappa Hospitals, Sri Devaraj Urs Medical College and University and the Department of Medicine for their support.

\section{References}

[1] Aydin Y, Berker D, Ustun I, Balcik O, Balli DN, Delibasi T, Peksoy I \& Guler S (2008), A very rare case of aplastic anemia: Grave's Disease. Southern Medical Journal 101, 666-667.

[2] Biswas N, Ahn YH, Goldman JM \& Schwartz JM (1991), Aplastic anemia associated with antithyroid drugs. American Journal of Medical Sciences 301, 190-194.

[3] Escobar-Morreale HF, Bravo P, Garcia-Robles R, Garcia-Laran a J, de la Calle H \& Sancho JM (1997), Methimazole-induced severe aplastic anemia: unsuccessful treatment with recombinant human granulocyte-monocyte colony stimulating factor. Thyroid 7, 67-70.

[4] Kumar M \& Goldman J (2002), Severe aplastic anemia and Grave's disease in a paediatric patient. British Journal of Haematology 118 , 327.

[5] Richardson JS, Sarkany I \& Campbell CD (1954), Fatal case of marrow aplasia after treatment with carbimazole. British Medical Journal $1,364-365$.

[6] The International Agranulocytosis and Aplastic Anemia Study (1987), Incidence of aplastic anemia: the relevant diagnostic criteria. Blood 70 1718.

[7] Williams DM (1999), Pancytopenia, aplastic anemia and pure red cell aplasia (GR Lee, J Foerster, J Lukens, et al. ed), Williams \& Wilkins, Baltimore, pp. 1186-1187.

[8] Young NS, Calado RT \& Scheinberg P (2006), Current concepts in the pathophysiology and treatment of aplastic anemia. Blood 108, 250. 